\title{
Why is right-wing media consumption associated with lower compliance with COVID-19 measures?
}

\author{
Vladimir Ponizovskiy ${ }^{1}$, Lusine Grigoryan ${ }^{2}$, and Wilhelm Hofmann ${ }^{2}$ \\ ${ }^{1}$ Jacobs University Bremen, Bremen International Graduate School of Social Sciences (BIGSSS) \\ ${ }^{2}$ Ruhr University Bochum, Faculty of Psychology
}

\begin{abstract}
Exposure to right-wing media has been shown to relate to lower perceived threat from COVID19 , lower compliance with prophylactic measures against it, and higher incidence of infection and death. What features of right-wing media messages account for these effects? In a preregistered cross-sectional study $(N=554)$ we test a model that differentiates perceived consequences of two CDC recommendations-washing hands and staying home-for basic human values. People who consumed more right-wing media perceived these behaviors as less beneficial for their personal security, for the well-being of close ones, and the well-being of society at large. Perceived consequences of following the CDC recommendations mediated the relationship between media consumption and compliance with recommendations. Implications for public health messaging are discussed.
\end{abstract}

Keywords: political ideology, media, value-instantiating beliefs, COVID-19, compliance

COVID-19 poses an unprecedented challenge to public health systems worldwide. The lack of available vaccine and failure to contain the virus make the outcomes of this pandemic particularly dependent on behavioral choices of individuals, such as decisions to comply or not comply with the recommendations of health authorities. Understanding the mechanisms that affect decisions to comply with public health recommendations is important for limiting the harm caused by COVID-19.

Some predictors of compliance with health recommendations are well-established, such as trust in the institutions, income, and gender (Bish \& Michie, 2010). The COVID-19 pandemic, however, is further complicated by the politization of the virus in many countries. In the US, party affiliation of public officials is associated with differences in frequency and content of messaging on COVID-19: Democratic politicians discussed the pandemic more frequently and placed more focus on public health risks than the Republicans, and the Republicans discussed economic implications of the virus and China's role in its origins more frequently (Green et al., 2020). Partisan media similarly presented American viewers with conflicting messages (Bursztyn et al., 2020, Motta et al., 2020). Republican party affiliation and exposure to right-wing media have been shown to relate to lower compliance with $\mathrm{CDC}$ health recommendations regarding social distancing (Allcott et al., 2020, Andersen, 2020, Barrios \& Hochberg, 2020; Simonov et al., 2020; Wright

Correspondence should be addressed to Vladimir Ponizovskiy, 309 South Hall, Campus Ring 1, Bremen, Germany, 28759. Email: vladimir.ponizovskiy@gmail.com et al., 2020), lower perceptions of risks associated with COVID-19 (Allcott et al., 2020; Barrios \& Hochberg, 2020), and lower fear of the virus (Harper et al., 2020). Even more alarmingly, exposure to right-wing media has been associated with higher infection rates and death rates for COVID-19 (Bursztyn et al., 2020).

Understanding the psychological mechanisms through which exposure to right-wing political messaging affects compliance with public health recommendations on COVID-19 can help a) identify the features of messages that can cause harm to public health, b) communicate to the media the reasons for avoiding such features in their messaging, and c) develop strategies for mitigating and counteracting the harm done by politicized messaging.

\section{Information and Attitude Formation}

The Theory of Planned Behavior (TPB; Ajzen, 1991; Ajzen \& Fishbein, 1975) provides a conceptualization of the effects of exposure to information, such as mass media messages, on attitudes and behavior. Ajzen and Fishbein (1975) proposed that attitudes are a product of knowledge about the attributes of an object or a behavior and subjective evaluations of these attributes. In this model, "a person is viewed as processing the information he has about an object in arriving at his evaluation of the object" (Ajzen \& Fishbein, 1975, p. 222). Differences in available information about an object can therefore lead to differences in attitudes.

This intuitive model of attitude formation is commonly shared among psychologists, but using it to predict attitudes and behavior from information has proved difficult. TPB posits a number of boundary conditions to the effects of information on the attitude: to affect the attitude, information has to be relevant, ac- 
cepted (believed), and non-redundant; even if all these conditions are satisfied, the size of the effect would depend on subjective evaluation of the relevant attribute (Ajzen \& Fishbein, 1975). The boundary conditions are numerous and latent, therefore difficult to assess.

The elusiveness of the information-behavior link is illustrated by research findings. Research in health communications shows that even when the information is purposefully designed to produce change in behavior, this effect is typically small (Abroms \& Maibach, 2008; Noar, 2006, Snyder \& Hamilton, 2002). While in some cases knowledge about health conditions is related to health behaviors (e.g., Lael-Monfared et al., 2019; Lindau et al., 2002, Osborn et al., 2011), studies often find weak or non-significant relations between the two constructs (e.g., Chen et al., 2014, Guerra et al., 2005; Hwang et al., 2014) (see Berkman et al., 2011, for a relevant review). In a series of studies, Ajzen (2011) found no relationship between seemingly relevant knowledge and behavior: environmental knowledge was unrelated to conservation behavior, and alcohol knowledge to drinking behavior. Ajzen concludes that evaluative implications of information-rather than its mere availability_affect behavior. It is therefore particularly helpful to identify features of messages about COVID-19 that have evaluative implications and that are likely to induce attitudinal and behavioral change (Van Bavel et al., 2020).

\section{Value-Instantiating Beliefs}

The Ajzen and Fishbein's model (Ajzen \& Fishbein, 1975) posits that any object or behavior can be evaluated according to an unlimited number of attributes, but only a small subset of such evaluations will contribute to the attitude. Assessing and comparing grounds for evaluation necessitates a comprehensive typology of such grounds; TPB does not provide it. We propose using Schwartz's (Schwartz, 1992) typology of values to assess perceived implications of objects and events. Values, as defined by Schwartz (1992), are beliefs about desirable end-states that serve as grounds for evaluation and selection of objects, events, and behaviors, making them well-suited for the role. Table 1 provides the list of values and their definitions.

We introduce the construct of value-instantiating beliefs (VIBs) to describe personal beliefs about perceived consequences of objects, actions, or events for values (Ponizovskiy et al., 2019; Ponizovskiy, 2020). Examples of VIBs are "COVID-19 is dangerous" (positing a negative relationship between an object, COVID-19, and the value of security) or "social distancing limits my freedom" (positing a negative relationship between the behavior, social distancing, and the value of self-direction). VIBs may differ in strength (COVID-19 can be seen as very, moderately, or slightly dangerous) and in direction (social distancing can be seen as limiting or expanding personal freedom). VIBs
Table 1

Definitions and examples of basic human values.

\begin{tabular}{ll}
\hline Definition & Example \\
\hline Security & $\begin{array}{l}\text { Safety, harmony, and stability of soci- } \\
\text { ety, of relationships, and of self }\end{array}$ \\
Conformity & $\begin{array}{l}\text { Restraint of actions, inclinations, and } \\
\text { impulses likely to upset or harm oth- } \\
\text { ers and violate social expectations or } \\
\text { norms }\end{array}$
\end{tabular}

Tradition Respect, commitment, and acceptance of the customs and ideas that traditional culture or religion provide

Benevolence Preservation and enhancement of the welfare of people with whom one is in frequent personal contact

Universalism Understanding, appreciation, tolerance, and protection for the welfare of all people and for nature

Self-direction Independent thought and actionchoosing, creating, exploring

Stimulation Excitement, novelty, and challenge in life

Hedonism Pleasure and sensuous gratification for oneself

Achievement Personal success through demonstrating competence according to social standards

Power Social status and prestige, control or dominance over people and resources

Note. Adapted with permission from Schwartz, 1994. (C) John Wiley and Sons.

can be acquired through socialization and may moderate the relationship between socialization experiences and attitudes (Ponizovskiy et al., 2019, Ponizovskiy, 2020).

VIBs are beliefs that have evaluative implications, as proposed by Ajzen (2011), and can capture the differences in perceptions of measures to combat COVID-19 that arise from differences in exposure to right- vs. left-wing media. In addition, they may provide insight into the content of such differences: Perceived consequences for which values differ between consumers of right- vs. left-wing media? Which of these perceived consequences are relevant to attitudes 
towards preventative measures and to compliance with them?

\section{Current Study}

In this study, we test a value-based model of attitudes toward and compliance with two CDC recommendations for COVID-19: staying home (Centers for Disease Control and Prevention, 2020a) and washing hands often (Centers for Disease Control and Prevention, 2020b). We hypothesize that right-wing media-as compared to left-wing media-provide consumers with information that downplays the positive consequences of complying with the CDC recommendations for basic human values, such as security, benevolence, and self-direction. Consumers of rightwing media, after being exposed to this information, form less positive VIBs about these behaviors, which, in line with Ajzen and Fishbein's (1975) Expectancy Value Theory, jointly contribute to less positive attitude towards and lower compliance with the recommendations.

We developed and preregistered the following set of hypotheses for the study:

H1: Exposure to right-wing media will be associated with weaker compliance with the CDC recommendations. Specifically:

H1a: Exposure to right-wing media will be associated with leaving one's residence more often.

$\mathrm{H} 1 \mathrm{~b}$ : Exposure to right-wing media will be associated with washing one's hands less often.

H2: Relationship between exposure to right-wing media and attitudes towards following the CDC recommendations will be mediated by VIBs. Specifically:

H2a: VIBs will mediate the relationship between exposure to right-wing media and attitude towards leaving one's residence.

$\mathrm{H} 2 \mathrm{~b}$ : VIBs will mediate the relationship between exposure to right-wing media and attitude towards washing hands.

The theoretical model is summarized in Figure 1 .

To test these hypotheses, we conducted a large online survey measuring media exposure, VIBs, attitudes, and self-reported compliance with health officials' recommendations and fit the data to the theoretical model presented above. Additionally, we compared our model to competing models. The first competing model considered perceived efficacy of CDC recommendations instead of VIBs as the mediator of the media-attitude relation. Perceived efficacy is commonly used to capture subjective assessments of policies in health communications (Rogers, 1975, 1983). The second competing model used political orientation instead of media exposure to predict VIBs. While media exposure is a more proximal indicator of informational input, political affiliation can affect broader patterns of information consumption through selective exposure, or preference for messages that are in line with one's political views /autociteKnoblochWesterwick2009, Stroud2010.

\section{Method}

\section{Sample}

The required sample size was estimated using a simulation study. The $\mathrm{R}$ code of the simulation study, as well as all study materials and data can be found on the OSF platform: https://osf.io/ft4q3/?view_only= 1f1185ef50414bffa77f6a0e6078fd30. The goal was to obtain .95 power to detect the smallest effect of interest (indirect effect of media exposure on the attitude towards following CDC recommendations) of .20 at the .05 alpha error probability. The target sample size was 550. All residents of the United States over 18 years old were eligible to participate. Participants who were required by their employers to travel to work were screened from the study. The effective sample size was 553 participants.

Forty-eight U.S. states were represented in the sample, except for Idaho and Wyoming. Sixty-two percent of participants were male, $36.5 \%$ were female, and $0.1 \%$ identified as "other". The mean age was 37.3 years $(\mathrm{SD}=10.4)$. Seventy-two percent completed bachelor's degree or higher. The average number of people in the household was 2.94 (SD = 1.35). Fiftysix percent of participants said they lived in a big city or a suburb of a big city, while $43.5 \%$ said they lived in a town/small city, in a village, or on a farm. Thirtyeight percent of participants reported household income of less than $\$ 50,000$, and $61.6 \%$ reported higher income. On the political orientation scale, $39.2 \%$ of participants described themselves as leaning left and $45.9 \%$ - as leaning right.

\section{Procedure and Measures}

Participants were recruited via the Amazon Mechanical Turk services and were reimbursed 1.50 USD for participation in the survey. The data was collected on May 12, 2020. All participants provided informed consent. The study received ethical approval from the University's ethics committee. The survey started with questions about behavior and attitudes, followed with a VIB questionnaire for the behavior of staying home. Then participants reported on their socio-demographic background and media consumption and completed the second VIB questionnaire for the behavior of washing hands. The average completion time was $6.5 \mathrm{~min}-$ utes.

\section{Measures}

Behavior. Staying home compliance was measured with the following question: "How many times during the past week did you leave your residence? Try to remember as accurately as possible. Please count 
Figure 1

Theoretical model: Predicting compliance behavior from exposure to right-wing media through VIBs and attitude

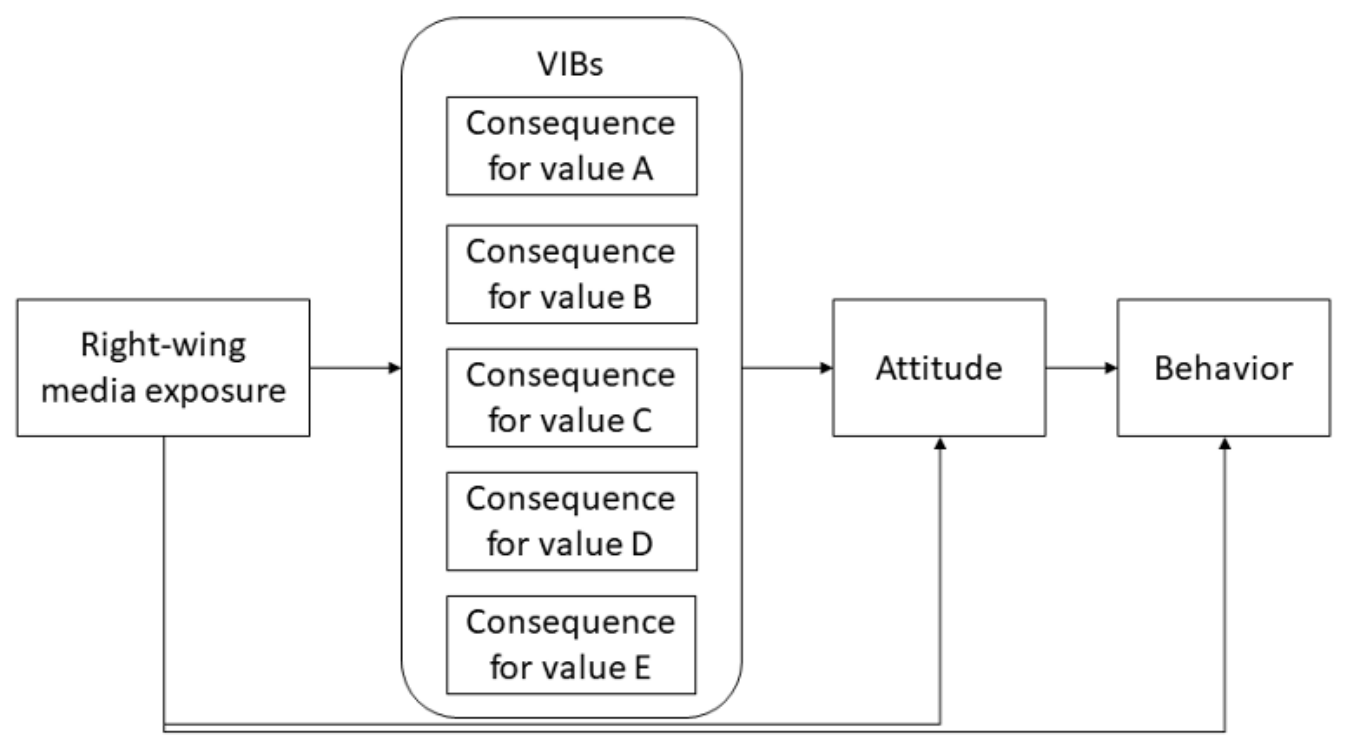

the times when you left your residence for more than 15 minutes. Do not include going to the yard or throwing out the trash." Participants were asked to input a number. Hand washing compliance was measured with the question: "How many times have you washed your hands yesterday? Try to remember as accurately as possible." Participants were asked to choose between six options: $1=$ "0-1 times", $2=$ "2-4 times", 3 = "5-7 times", 4 = "8-10 times", 5 = "11-13 times", $6=$ "14 times or more". While the distribution of hand washing was close to normal $(M=3.4, S D=1.3$, skewness $=0.43$, kurtosis $=-0.54)$, the distribution of leaving home strongly deviated from normal $(M=3.7, S D$ $=4.6$, skewness $=4.54$, kurtosis $=32.59$ ). Ten percent of all participants did not leave their homes during the week, and 55\% left home between one and three times. We therefore deviated from the preregistration protocol and transformed this variable by taking a square root of the reported number of outings. The distribution of the transformed variable was close to normal $(M=1.67$, $S D=0.97$, skewness $=0.96$, kurtosis $=3.42$ ).

Attitudes. Attitudes were measured with a 5item, 7-point $(-3$ to +3$)$ semantic differential scale with the prompts "Staying at home during the pandemic is..." and "Washing my hands more often during the pandemic is..." and anchors "good-bad", "harmful-beneficial", "pleasant-unpleasant", "uselessuseful", and "enjoyable-unenjoyable". Alpha reliability was .83 for the attitude towards staying home and .78 for the attitude towards washing hands.

Value-instantiating beliefs. VIBs were measured with the 21-item VIBs Questionnaire (Anonymized, 2020). Each item represents a semantic differential scale that elicits evaluations of consequences of one behavior for one value. The questionnaire covers consequences of behaviors for all ten values from the original version of Schwartz theory of basic values (Schwartz, 1992): self-direction, stimulation, hedonism, achievement, power, security, conformity, tradition, benevolence, and universalism. For example, a conformity VIB anchors were: [A behavior would] "Violate the rules and expectations of others" versus "Be in line with the rules and expectations of others", a 7-point scale, from -3 to +3 . The behavioral prompts were "Staying at home during the pandemic would..." and "Washing my hands more often during the pandemic would...". Mean standardized alpha reliability was .64 for VIBs of staying home and .67 for VIBs of washing hands 1 .

Media Exposure. Media exposure was measured with the matrix question: "How often do you get your news from the following outlets?" with rows "Fox news", “CNN", "MSNBC”, "NPR”, "New York Times", "Washington Post", "Sean Hannity (radio)", "Rush Limbaugh (radio)", "Breitbart", and a 5-point Likert-type response scale ( 1 = "Never", 5 = "Very often").

Perceived efficacy of CDC recommendations. "In your opinion, how effective are the following recommendations at combating the spread of COVID19?" with items "Staying at home" and "Washing your hands" and a 7-point Likert-type response scale, from $1=$ "Not effective at all" to 7 = "Extremely effective".

\footnotetext{
${ }^{1}$ We initially planned to center VIB scores within individuals to correct for individual response tendency. However, it became apparent that this procedure is statistically redundant when multiple VIBs are included in the model. We therefore used the raw scores.
} 
Sociodemographic variables. Participants reported whether they are required to travel to work (exclusion criterion), their age, gender, education, number of people in the household, state of residence, place of residence (settlement size), objective and subjective income, and political orientation. A detailed report on these measures is available on the project's OSF page.

\section{State-Level Data}

To control for environmental factors that might affect compliance behavior, we used secondary statelevel data. The number of COVID-19 infections and deaths per state were obtained from CDC (CDC, 2020c) and information on state-level shelter-in-place policies on the date of data collection was obtained from the COVID-19 US State Policy Database (Raifman et al., 2020).

\section{Results}

In line with the preregistered analysis plan, we first tested which VIBs were most relevant for each attitude. For attitude towards staying home, the strongest correlations were obtained for hedonism $(r(551)=.51$, $p<.001)$, security $(r(551)=.47, p<.001)$, selfdirection $(r(551)=.42, p<.001)$, universalism $(r(551)$ $=.42, p<.001)$, and benevolence $(r(551)=.41, p<$ $.001)$ VIBs. For attitude towards washing hands, the strongest correlations were obtained for universalism $(r(551)=.48, p<.001)$, benevolence $(r(551)=.44, p$ $<.001)$, hedonism $(r(551)=.41, p<.001)$, security $(r(551)=.40, p<.001)$, and conformity $(r(551)=.37$, $p<.001)$ VIBs.

We further examined the measurement models for the latent variables of attitudes and media exposure. Attitudes towards staying home and washing hands were tested in a single model as correlated factors. This model required one modification: a covariance was added between the items "pleasant-unpleasant" and "enjoyable-unenjoyable" for both attitudes. The resulting model had a good fit (CFI $=.946$; RMSEA $=.096, \mathrm{SRMR}=.008)$. The two attitudes correlated positively ( $r=.76, p<.001)$. Exposure to right- and left-wing media was also tested in a single model with correlated latent constructs. Right-wing media were represented by Fox news, Sean Hannity (radio), Rush Limbaugh (radio), and Breitbart. Left-wing media were represented by CNN, MSNBC, NPR, New York Times, and Washington Post. The model showed good fit without any modifications $(\mathrm{CFI}=.970, \mathrm{RMSEA}=$ .081$, SRMR $=.044)$. The two factors correlated positively $(r=.53, p<.001)$. See Online Supplement for details.

In the next step, we tested two structural equation models (SEM) as specified in the preregistration: media exposure predicted behaviors of staying home and washing hands through VIBs and attitudes (Fig. 1). In both models, we controlled for age, gender, education, political orientation, subjective income, objective income, number of people in the household, place of residence, and state-level number of infections, deaths, and shelter-in-place policies. We deviated from the preregistered protocol by adding a latent construct for left-wing media exposure: otherwise, right-wing media exposure was confounded with total amount of media consumption, as indicated by an $r=.53$ correlation between the two types of media consumption.

\section{Compliance with "staying home" recommendation}

The initial model showed sub-optimal fit to the data $(\mathrm{CFI}=0.861, \mathrm{RMSEA}=.07, \mathrm{SRMR}=.099)$. None of the control variables, except the number of COVID19 cases in the state, predicted behavior. We therefore simplified the model by removing all controls except for the number of cases. The modified model showed good fit to the data (CFI $=.940$, RMSEA $=.065$, SRMR $=.081)$. The number of state-level Covid-19 cases did not predict behavior in the modified model $(b=.03, \mathrm{SE}=.04, p=.412)$. Figure 2 presents the unstandardized regression coefficients for all variables in the model, except controls.

As predicted (H1a), exposure to right-wing media was related to leaving home more often $(b=.21,95 \%$ CI $[.10, .31], \mathrm{SE}=.05, p<.001)$. The total effect of right-wing media exposure on behavior through both direct and indirect paths was $b=.27, \mathrm{SE}=.05, p<$ .001 . The model explained $8 \%$ of variance in behavior. Also as predicted $(\mathrm{H} 2 \mathrm{a})$, the effect of right-wing media on attitude towards staying home was mediated be VIBs. Indirect effects of exposure to right-wing media on attitude were significant via security $(b=-.13$, $95 \%$ CI $[-.19,-.07], \mathrm{SE}=.03, p<.001)$, hedonism $(b=.07,95 \%$ CI $[.03, .11], \mathrm{SE}=.02, p<.001)$, and universalism $(b=-.05,95 \%$ CI $[-.09,-.01], \mathrm{SE}=.02$, $p=.016)$ VIBs. The total effect of right-wing media exposure on attitude through both direct and indirect paths was $b=-.45,95 \%$ CI $[-.57,-.32], \mathrm{SE}=.06, p<$ 001 . The model explained $46 \%$ of variance in attitude towards staying home ${ }^{2}$.

\section{Compliance with "washing hands often" recom- mendation}

The initial model showed sub-optimal fit (CFI = .855$, RMSEA $=.073$, SRMR $=.110)$. Only two of the controls were significantly related to behavior. People with higher objective income $(b=.18, \mathrm{SE}=.07, p=$ $.011)$ and women $(b=.29, \mathrm{SE}=.11, p=.010)$ reported higher frequency of washing hands. After simplifying the model by removing non-significant controls, model showed a good fit to the data $(\mathrm{CFI}=.930, \mathrm{RMSEA}=$

\footnotetext{
${ }^{2}$ The results were essentially identical in the models with and without all control variables. Additionally, we tested the model with the raw scores for behavior using a negative binomial SEM for robustness check. The findings remained unchanged. These additional analyses are reported in the Online Supplement.
} 
Figure 2

Structural equation model predicting compliance with "staying home" recommendation from media exposure, VIBs, and attitudes

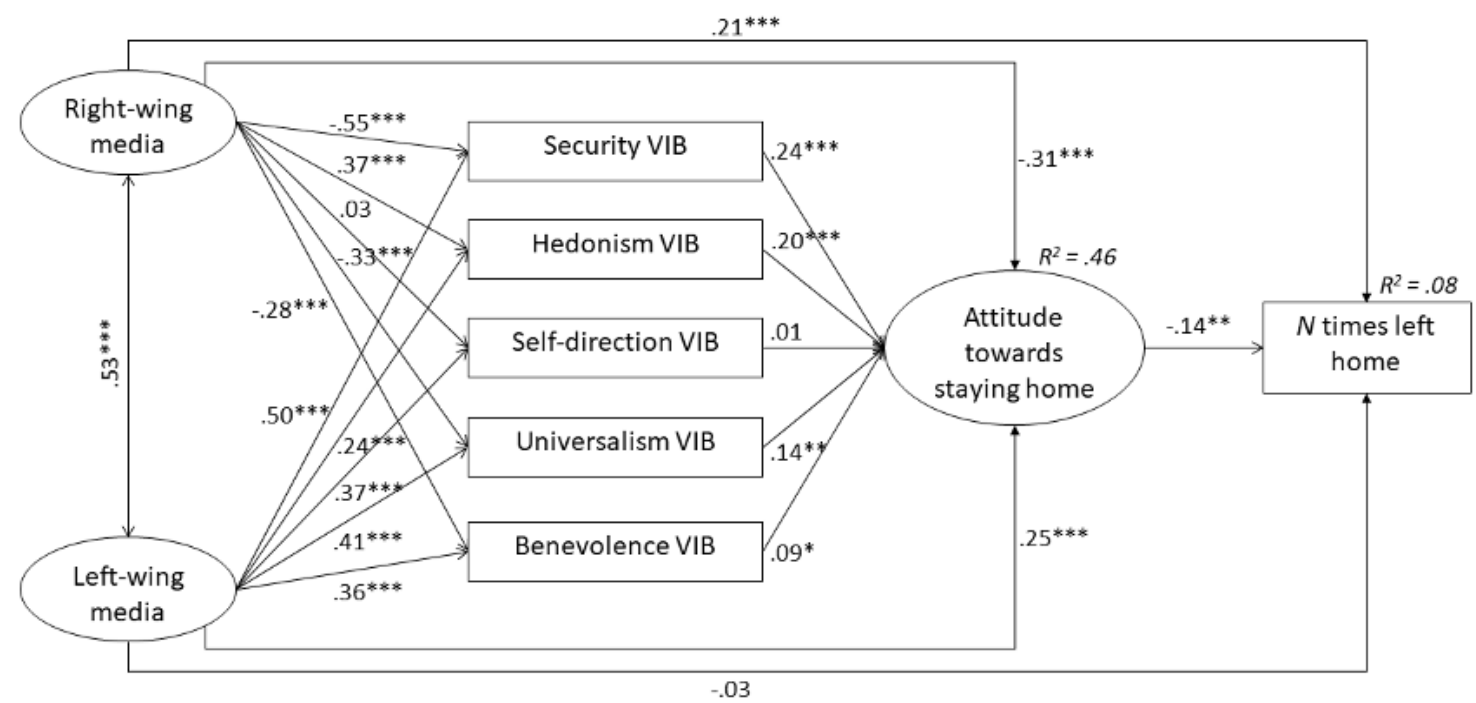

Note. Note. ${ }^{*} \mathrm{p}<.05, * * \mathrm{p}<.01, * * * \mathrm{p}<.001$. Unstandardized regression coefficients. $\mathrm{N}=553$.

$.067, \mathrm{SRMR}=.099)$. The effects of objective income $(b=.13, \mathrm{SE}=.06, p=.028)$ and gender $(b=.29, \mathrm{SE}$ $=.11, p=.007)$ remained significant in the modified model. Figure 3 presents the unstandardized regression coefficients for all variables in the model, except controls.

The effect of right-wing media on the frequency of washing hands was fully mediated by VIBs and attitudes (total indirect effect: $b=-.14,95 \%$ CI [-.21, $.07], \mathrm{SE}=.04, p<.001)$. The direct $(b=.13,95 \%$ CI $[-.02, .28], \mathrm{SE}=.08, p=.09)$ and total $(b=-.01$, $95 \%$ CI $[-.15, .13], \mathrm{SE}=.07, p=.908)$ effects were not significant, providing only partial support for H1b. The model predicted $7 \%$ of variance in behavior. As predicted $(\mathrm{H} 2 \mathrm{~b})$, the effect of right-wing media on attitude towards washing hands was mediated by VIBs. Indirect effects of exposure to right-wing media on attitude were significant via security $(b=-.07,95 \% \mathrm{CI}$ $[-.11,-.03], \mathrm{SE}=.02, p=.001)$, benevolence $(b=-.05$, $95 \% \mathrm{CI}[-.08,-.01], \mathrm{SE}=.02, p=.006)$, and hedonism $(b=.04,95 \%$ CI $[.01, .07], \mathrm{SE}=.01, p=.006)$ VIBs. The total effect of right-wing media exposure on attitude through direct and indirect paths was $b=-.44$, $95 \%$ CI $[-.54,-.33], \mathrm{SE}=.05, p<001$. The model explained $43 \%$ of variance in attitude towards washing hands.

\section{Competing models}

We further compared our models with an alternative set of models, where this relationship was mediated by perceived efficacy of CDC recommendations. The model predicting compliance with recommendation to stay home from media, perceived efficacy of this recommendation, and attitude, showed a good fit to the data $(\mathrm{CFI}=.959, \mathrm{RMSEA}=.059, \mathrm{SRMR}=.078)$. Perceived efficacy outperformed VIBs in predicting attitude towards staying home $\left(\mathrm{R}^{2}=.62 \mathrm{vs}\right.$. $\left.\mathrm{R}^{2}=.46\right)$. The indirect effect of media exposure on attitude via efficacy was $b=-.31, \mathrm{SE}=.04, p<.001$, and the indirect effect on behavior was $b=.04, \mathrm{SE}=.01, p=.005$. The model predicting frequency of washing hands also showed acceptable fit to the data (CFI $=.935$, RMSEA $=.068$, SRMR $=.105)$. In this case, the model with VIBs outperformed the model with perceived efficacy as a mediator: the indirect effects of media on attitude and behavior via efficacy were not significant $(b=-.03$, $\mathrm{SE}=.03, p=.217$ and $b=-.01, \mathrm{SE}=.01, p=.235$, respectively). Both models explained approximately $43 \%$ of variance in attitudes.

To examine whether media exposure is a better predictor of compliance than political orientation, we tested competing models where media exposure was replaced with political orientation. Both models with political orientation showed acceptable fit to the data $(\mathrm{CFI}=.916, \mathrm{RMSEA}=.098, \mathrm{SRMR}=.082$ for staying home, CFI $=.922$, RMSEA $=.087$, SRMR $=$ .082 for washing hands). For staying home, the model with political orientation explained less variance in attitude (44\% vs. $46 \%$ ) and behavior (5\% vs. $8 \%$ ) than the original model with media exposure. For washing hands, the model with political orientation explained less variance in attitude (39\% vs. $43 \%)$ and the same amount of variance in behavior (7\%) compared to the original model with media exposure.

\section{Discussion}

In this study, we estimated a value-based model of attitudes toward and compliance with CDC recommen- 
Figure 3

Structural equation model predicting compliance with "washing hands often" recommendation from media exposure, VIBs, and attitudes

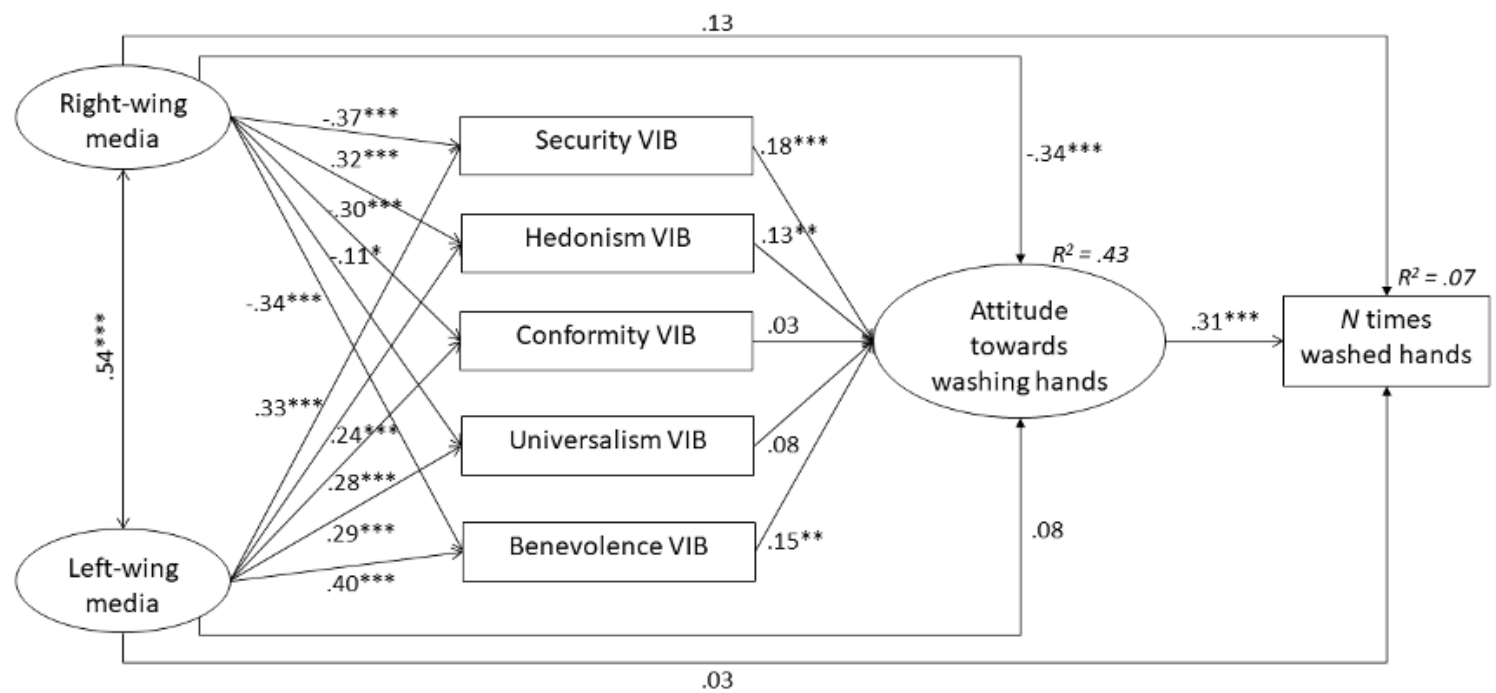

Note. Note. ${ }^{*} \mathrm{p}<.05, * * \mathrm{p}<.01, * * * \mathrm{p}<.001$. Unstandardized regression coefficients. $\mathrm{N}=553$.

dations to understand what features of right-wing messaging on COVID-19 might explain lower compliance. We found that people who consume right-wing media perceive following $\mathrm{CDC}$ recommendations as having less positive consequences for the values of security, benevolence, and universalism, and that these differences are associated with less positive attitudes and lower compliance. For staying at home, exposure to right-wing media related to lower frequency of the behavior both directly and indirectly. For washing hands, exposure to right-wing media only related to lower frequency of the behavior indirectly, through VIBs and attitudes. Our findings concur with other studies that showed a link between exposure to right-wing media and compliance with health officials' recommendations on COVID-19 (Allcott et al., 2020, Andersen, 2020, Barrios \& Hochberg, 2020, Simonov et al., 2020; Wright et al., 2020).

\section{The "Why?" of the media-behavior link}

While exposure to right-wing media affected almost all VIBs, only some of these VIBs further translated into the attitude and behavior. Unsurprisingly, the strongest mediating effect was for the security VIB: The more people consumed right-wing media, the less positively they perceived the consequences of staying home and washing hands for their security.

A weaker, but comparable in size mediating effect was observed for prosocial VIBs: perceived consequences for benevolence (concern for the well-being of close others) and universalism (concern for the wellbeing of all people). Benevolence, but not universalism VIB mediated the media-attitude relationship for washing hands. Universalism, but not benevolence
VIB mediated the media-attitude relationship for staying at home. This may relate to the functions of the behaviors as understood by the participants: whereas washing hands limits the transmission of the virus in one's immediate surroundings (and is therefore most relevant to the health of close others), social distancing is portrayed as a way to "flatten the curve", or reduce the burden of COVID-19 on society in general.

Unexpectedly, the media-behavior link was also mediated by hedonism VIB (perceived consequences of behaviors for sensual pleasure), for both behaviors. Hedonism was also the only VIB that was predicted positively by both right-wing and left-wing media consumption; for all others, the links with two types of media were in opposite directions. We have no clear interpretation of the finding but suspect that people who consume more media in general may find more pleasure in the everyday routine.

\section{Alternative explanations}

The proposed model where media consumption predicted attitudes and behavior outperformed an alternative model where attitudes and behavior were predicted from political orientation. When both media consumption and political orientation were in the model, only media consumption predicted behavior. This finding suggests that media is a more proximal predictor of attitudes and behavior, in line with the informationbased account of attitude formation (Ajzen \& Fishbein, 1975).

Using perceived efficacy of CDC recommendations as a mediator instead of VIBs showed mixed results. While efficacy predicted more variance in attitude towards staying home compared to VIBs, it did not me- 
diate the relationship between media consumption and attitude towards washing hands often. VIBs were more consistent as mediators of media-attitude link across different behaviors. There are important differences between perceived efficacy and VIBs as grounds for judgments: Perceived efficacy is more specific in that it only informs us about one important belief; VIBs are more abstract in that they inform us about a range of different beliefs relevant to humans' evolutionary needs. The presented data does not allow for definitive conclusions, but VIBs might have an advantage over perceived efficacy in particular use cases. VIBs capture a wider range of beliefs relevant to an attitude and can provide information about relative contributions of qualitatively different beliefs to the attitude.

\section{Limitations}

First, the study is cross-sectional and does not establish causality. However, the causal link between rightwing media consumption and compliance with health officials' recommendations for combatting COVID-19 was demonstrated in earlier studies (Bursztyn et al., 2020; Simonov et al., 2020, Wright et al., 2020). The goal of the current study was to examine the psychological mechanism linking media consumption to behavior. Second, although our model explained over $40 \%$ of variance in attitudes towards both behaviors, it accounted only for about $7-8 \%$ of variance in actual behavior. Attitude was only weakly related to behavior in both cases. A longitudinal study tracking changes in behavior instead of a one-shot measure of average behavior during a week (leaving home) or a day (washing hands) might have shown stronger effects. We would argue that despite the small proportion of explained variance in behavior, these findings can have large implications for slowing down the spread of the virus. First, even small changes in behavior can affect the speed of transmission. Second, changes in attitudes can affect behavior and transmission rates in the long run by shaping the normative climate (Van Bavel et al., 2020).

\section{Implications}

In this study, we identified differences in beliefs that are relevant to the link between exposure to right-wing media and lower compliance with CDC recommendations for COVID-19. Our findings suggest that utmost attention should be given to accurate representations of health risks associated with COVID-19 in the media, and that highlighting consequences of following prophylactic measures for the well-being of others has the potential to affect public health behavior during COVID-19 pandemic.

Previous research found that right-wing politicians (Green et al., 2020) and media outlets (Motta et al., 2020) downplay the public health risks associated with COVID-19. In our data, beliefs about consequences of prophylactic measures for personal security were the strongest mediators between media consumption and compliance with CDC recommendations. These results highlight the strength of behavioral consequences that can follow the (mis)representation of public health risks of COVID-19. These findings also contradict the suggestion that overly grim representations of COVID19 can induce the feeling of helplessness and be counterproductive (Van Bavel et al., 2020) - in our data, the more relevant to their personal security participants found prophylactic measures, the more they followed the prophylactic protocol.

The comparable size of the effects of pro-social VIBs (benevolence and universalism) suggests that compliance with CDC recommendations is also affected by the portrayal of prophylactic measures in terms of their consequences for others. This finding corroborates early accounts of effectiveness of prosocial messaging on health behavior during the COVID19 crisis (Brooks et al., 2020, Everett et al., 2020). Additionally, in our data, prosocial VIBs only played a role when they were congruent with the function of the behavior: Perceptions of consequences for benevolence mediated the media-behavior relation for the behavior that primarily affects people in the same household (washing hands), and perceived consequences for universalism mediated the effect for the behavior that affects general transmission (staying home). This underscores the importance of messaging that is congruent with people's motivations.

\section{References}

Abroms, L. C., \& Maibach, E. W. (2008). The Effectiveness of Mass Communication to Change Public Behavior. Annual Review of Public Health, 29(1), 219-234. https://doi.org/10. 1146/annurev.publhealth.29.020907.090824

Ajzen, I. (1991). The theory of planned behavior. In Handbook of theories of social psychology (pp. 179-211). https :// doi . org/10.4135/ 9781446249215.n22

Ajzen, I., \& Fishbein, M. (1975). Belief, attitude, intention and behavior: An introduction to theory and research. Reading, MA, AddisonWesley. https://doi.org/10.2307/2065853

Ajzen, I., Joyce, N., Sheikh, S., \& Cote, N. G. (2011). Knowledge and the prediction of behavior: The role of information accuracy in the theory of planned behavior. Basic and Applied Social Psychology, 33(2), 101-117. https:// doi.org/10.1080/01973533.2011.568834

Allcott, H., York University, N., Research, M., Conway, J., Gentzkow, M., Thaler, M., \& Yang, D. (2020). Polarization and Public Health: Partisan Differences in Social Distancing during the Coronavirus Pandemic. https://doi. org/10.3386/w26946 
Andersen, M. (2020). Early Evidence on Social Distancing in Response to COVID-19 in the United States. SSRN Electronic Journal, (May). https://doi.org/10.2139/ssrn.3569368

Barrios, J. M., \& Hochberg, Y. V. (2020). Risk Perception Through the Lens of Politics in the Time of the COVID-19 Pandemic. SSRN Electronic Journal. https : / / doi . org / 10 . 2139/ ssrn . 3568766

Berkman, N. D., Donahue, K., Berkman, N. D., Sheridan, S. L., Donahue, K. E., Halpern, D. J., \& Crotty, K. (2011). Low Health Literacy and Health Outcomes: An Updated Systematic Review. Annals of Internal Medicine, 155(2). https://doi.org/10.1059/0003-4819-155-2201107190-00005

Bish, A., \& Michie, S. (2010). Demographic and attitudinal determinants of protective behaviours during a pandemic: A review. British Journal of Health Psychology, 15(4), 797-824. https: //doi.org/10.1348/135910710X485826

Brooks, S. K., Webster, R. K., Smith, L. E., Woodland, L., Wessely, S., Greenberg, N., \& Rubin, G. J. (2020). The psychological impact of quarantine and how to reduce it: rapid review of the evidence. The Lancet, 395(10227), 912-920. https ://doi .org/10.1016/S0140-6736(20) 30460-8

Bursztyn, L., Rao, A., Roth, C., \& Yanagizawa-Drott, D. (2020). Misinformation During a Pandemic. SSRN Electronic Journal. https://doi. org/10.2139/ssrn.3580487

Centers for Disease Control and Prevention. (2020a). Deciding to Go Out.

Centers for Disease Control and Prevention. (2020b). How to protect yourself \& others.

Chen, A. M., Yehle, K. S., Albert, N. M., Ferraro, K. F., Mason, H. L., Murawski, M. M., \& Plake, K. S. (2014). Relationships between health literacy and heart failure knowledge, selfefficacy, and self-care adherence. Research in Social and Administrative Pharmacy, 10(2), 378-386. https://doi.org/10.1016/j.sapharm. 2013.07.001

Everett, J. A., Colombatto, C., Chituc, V., Brady, W. J., \& Crockett, M. J. (2020). The effectiveness of moral messages on public health behavioral intentions during the COVID-19 pandemic. PsyArXiv, 1-23. https://doi.org/10.31234/ osf.io/9yqs8

Green, J., Edgerton, J., Naftel, D., Shoub, K., \& Cranmer, S. J. (2020). Elusive consensus: Polarization in elite communication on the COVID-19 pandemic. Science Advances, 6(28), eabc2717. https://doi.org/10.1126/ sciadv.abc2717

Guerra, C. E., Dominguez, F., \& Shea, J. A. (2005). Literacy and knowledge, attitudes, and behav- ior about colorectal cancer screening. Journal of Health Communication, 10(7), 651-663. https://doi.org/10.1080/10810730500267720

Harper, C. A., Satchell, L. P., Fido, D., \& Latzman, R. D. (2020). Functional Fear Predicts Public Health Compliance in the COVID-19 Pandemic. International Journal of Mental Health and Addiction. https://doi .org/10. 1007/s11469-020-00281-5

Hwang, B., Moser, D. K., \& Dracup, K. (2014). Knowledge is insufficient for self-care among heart failure patients with psychological distress. Health Psychology, 33(7), 588-596. https://doi.org/10.1037/a0033419

Lael-Monfared, E., Tehrani, H., Moghaddam, Z. E., Ferns, G. A., Tatari, M., \& Jafari, A. (2019). Health literacy, knowledge and self-care behaviors to take care of diabetic foot in low-income individuals: Application of extended parallel process model. Diabetes and Metabolic Syndrome: Clinical Research and Reviews, 13(2), 1535-1541. https://doi.org/ 10.1016/j.dsx.2019.03.008

Lindau, S. T., Tomori, C., Lyons, T., Langseth, L., Bennett, C. L., \& Garcia, P. (2002). The association of health literacy with cervical cancer prevention knowledge and health behaviors in a multiethnic cohort of women. American journal of obstetrics and gynecology, 186(5), 938-943. https://doi.org/10.1067/mob.2002. 122091

Motta, M., Stecula, D., \& Farhart, C. (2020). How Right-Leaning Media Coverage of COVID19 Facilitated the Spread of Misinformation in the Early Stages of the Pandemic in the U.S. Canadian Journal of Political Science. https://doi.org/10.1017/S0008423920000396

Noar, S. M. (2006). A 10-year retrospective of research in health mass media campaigns: Where do we go from here? Journal of Health Communication, 11(1), 21-42. https://doi.org/10. 1080/10810730500461059

Osborn, C. Y., Paasche-Orlow, M. K., Bailey, S. C., \& Wolf, M. S. (2011). The mechanisms linking health literacy to behavior and health status. American Journal of Health Behavior, 35(1), 118-128. https://doi.org/10.5993/AJHB.35. 1.11

Ponizovskiy, V., Grigoryan, L., Kühnen, U., \& Boehnke, K. (2019). Social construction of the value-behavior relation. Frontiers in Psychology, 10(APR). https://doi.org/10.3389/ fpsyg.2019.00934

Ponizovskiy, V. (2020). Value-instantiating beliefs: A practical substitute for meaning [https://psyarxiv.com/w7kns]. https : //doi.org/10.31234/osf.io/w7kns 
Rogers, R. W. (1975). A Protection Motivation Theory of Fear Appeals and Attitude Change1. The Journal of Psychology, 91(1), 93-114. https: //doi.org/10.1080/00223980.1975.9915803

Rogers, R. W. (1983). Cognitive and physiological processes in fear appeals and attitude change: A revised theory of protection motivation. In J. Cacioppo \& R. Petty (Eds.), Social psychophysiology (pp. 153-176). New York, Guilford Press.

Schwartz, S. H. (1992). Universals in the content and structure of values: Theoretical advances and empirical tests in 20 countries. Advances in experimental social psychology, 25(1), 1-65.

Simonov, A., Sacher, S., Dube, J.-P. H., \& Biswas, S. (2020). The Persuasive Effect of Fox News: Non-Compliance with Social Distancing During the COVID-19 Pandemic. SSRN Electronic Journal, (May). https:// doi .org/10. 2139/ssrn.3604214

Snyder, L., \& Hamilton, M. (2002). A meta-analysis of US health campaign effects on behav- ior: Emphasize enforcement, exposure, and new information, and beware the secular trend. In R. C. Hornik (Ed.), Public health communication: Evidence for behavior change (pp. 357-383). Mahwah, New Jersey, Lawrence Erlbaum Associates. https:// doi.org/10.1017/CBO9781107415324.004

Van Bavel, J. J., Baicker, K., Boggio, P. S., Capraro, V., Cichocka, A., Cikara, M., Crockett, M. J., Crum, A. J., Douglas, K. M., Druckman, J. N., Drury, J., Dube, O., Ellemers, N., Finkel, E. J., Fowler, J. H., Gelfand, M., Han, S., Haslam, S. A., Jetten, J., ... Willer, R. (2020). Using social and behavioural science to support COVID-19 pandemic response. Nature Human Behaviour, 4(5), 460-471. https://doi.org/10.1038/s41562-020-0884-z

Wright, A. L., Sonin, K., Driscoll, J., \& Wilson, J. (2020). Poverty and Economic Dislocation Reduce Compliance with COVID-19 Shelterin-Place Protocols. SSRN Electronic Journal. https://doi.org/10.2139/ssrn.3573637 